\title{
Survey of Knowledge, Attitudes and Practices of Brazilian Dentists Regarding Silver Diamine Fluoride
}

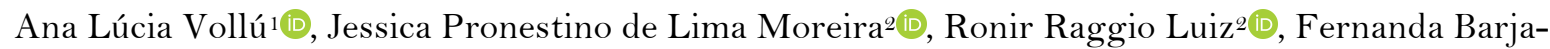 \\ Fidalgo $^{3}$ (i), Andrea Fonseca-Gonçalves ${ }^{1(0)}$
}

\begin{abstract}
${ }^{1}$ Department of Pediatric Dentistry and Orthodontics, School of Dentistry, Federal University of Rio de Janeiro, Rio de Janeiro, RJ, Brazil.

2Institute of Public Health Studies, Federal University of Rio de Janeiro, Rio de Janeiro, RJ, Brazil.

${ }^{3}$ Department of Preventive and Community Dentistry, School of Dentistry, State University of Rio de Janeiro, Rio de Janeiro, RJ, Brazil.
\end{abstract}

Author to whom correspondence should be addressed: Andréa Fonseca-Gonçalves, Departamento de Odontopediatria e Ortodontia, Faculdade de Odontologia, UFRJ, Rua Rodolpho Paulo Rocco, 325, Rio de Janeiro, RJ, Brazil. 21941-913. Phone: +55-21-3938-2098. E-mail: andrea.goncalves@odonto.ufrj.br.

Academic Editors: Alessandro Leite Cavalcanti and Wilton Wilney Nascimento Padilha

Received: 22 March 2019 / Accepted: 06 November 2019 / Published: 12 November 2019 How to cite this article: Vollú AL, Moreira JPL, Luiz RR, Barja-Fidalgo F, Fonseca-Gonçalves A. Survey of knowledge,
attitudes and practices of Brazilian dentists regarding silver diamine fluoride. Pesqui Bras Odontopediatria Clín Integr.
2020; 20:e4280. https://doi.org/10.1590/pboci.2020.014

\begin{abstract}
Objective: Current acceptability, barriers to use, and clinical/teaching practices of Silver Diamine Fluoride (SDF) among Brazilian dentists were investigated. Material and Methods: A Google forms questionnaire was sent to dentists $(n=10,500)$ to collect information about the use of SDF and the dentists' workplace, experience, specialty, and city of practice. A logistic regression model was performed. Results: From 409 respondents, $13.2 \%$ used SDF. Working at universities increased the use of SDF by 2.29 times $(\mathrm{p}=0.018)$ compared to private offices. Each training year, the chance of SDF usage increased by $3 \%(\mathrm{p}=0.008)$. Pediatric dentists are more likely to use SDF $(\mathrm{OR}=6.76, \mathrm{p}<0.001)$. There is no association between SDF usage and the city of practice. The majority (75.9\%) indicated SDF for noncompliant patients. Dentists $(75.9 \%)$ reported the exclusive use in deciduous teeth, while $24.1 \%$ also use in permanent teeth. The main barrier for non-users was a lack of knowledge (58.3\%), while for users, tooth staining (90.7\%) and parental acceptance (64.8\%) were the complaints. Conclusion: The Silver Diamine Fluoride is not a common product used by the dentists from RJ. Its clinical applicability should be further disseminated; thus, lack of scientific knowledge would cease to be a problem in the use of SDF to arrest caries lesions.

Keywords: Dental Caries; Cariostatic Agents; Dentists, Surveys and Questionnaires.
\end{abstract}




\section{Introduction}

Dental caries is the most common oral disease worldwide, affecting $60-90 \%$ of school children and nearly $100 \%$ of adults, often leading to pain and discomfort [1]. In Brazil, children of 5 years old present different decayed, missing, and filled teeth indices across the country, varying from 2.1 in the southeast region - where Rio de Janeiro State (RJ) is located - to 3.37 in the north. In addition, $80 \%$ and $54.1 \%$ of children at 5 and 12 years old, respectively, have decayed teeth, with operative treatment needs [2].

Access to dental care services is markedly low among people with low incomes, low education levels, and living in rural areas [1]. It is also difficult for people with special needs and young children [3]. According to new global oral health goals [4], arrest of dental caries, without using dental restoration, might meet the requirement of affordable cost, simplicity of implementation, and marked benefits.

Silver diamine fluoride (SDF) fulfills these requirements and can increase accessibility to oral health care because it is a low-cost, topical medicament, easily applicable, and used in many countries to arrest dental caries [5-7]. Although advocated since the 1960s, when formulated and approved in Japan, it has only recently taken a leading role in the world scene. In the USA, in August 2014, the Food and Drug Administration cleared the first SDF product for the market, making it available since April 2015 [7]. In Brazil, SDF is cleared to be used as a cariostatic and desensitizing agent. Thinking about public health, SDF can become an important tool in the management of caries lesions, avoiding pain and discomfort, either being used as a definitive or temporary treatment, mainly in places where the demand is great, with long queues of waiting.

Considering that substantial gaps exist in oral health outcomes, due to the fact that minorities, or those of low socioeconomic status, have reduced access to care, SDF promises to become an invaluable tool for low-cost and effective caries management [8]. Thus, knowing that the use of SDF was very widespread in the 1980s/1990s in Brazil, but fell into disuse, probably because of aesthetic concerns, the objective of this survey was to understand the current acceptability, barriers to use, and clinical and teaching practices of SDF among dentists registered in the RJ Regional Council of Dentistry (CRO-RJ).

\section{Material and Methods}

Study Design and Sample

A cross-sectional survey was conducted with 10,500 dentists of RJ, Brazil. All dentists from RJ, Brazil, active legally registered, and having an email contact in the CRO-RJ were invited to participate in the survey.

\section{Survey Instrument and Data Collection}

An online multiple-choice and fill-in response questionnaire was created in Google Forms. Questions comprised personal information, years of practice experience (graduation year), specialty, workplace, and city. Six questions covered the current use of SDF and investigated in which type of teeth and dentition it was used. Moreover, the application interval, clinical indications, and possible barriers to the use of SDF were also investigated. Finally, one issue, which was only to be answered by teachers, evaluated whether they taught their students about SDF. The questions that investigated the dentist's specialty, the clinical indication of SDF, and barriers to the use of SDF accepted more than one answer. The variables 'place of work' and 'city of performance' were categorized into private practice, public or military unit, and university; and capital, metropolitan area, and other cities, respectively.

The researchers contacted the CRO-RJ to inform them about the research objectives, and to request support in sending the survey invitation via email to all dentists in RJ. 
The email contained the following aspects: a brief explanation of the research objectives; the researchers' identification; and a link to access the questionnaire. Survey participants answered the questions by clicking on this link. The e-mail was fired twice, in March and May of 2017, to all registered dentists in RJ who had an active email contact in the CRO-RJ $(n=10,500)$.

\section{Data Analysis}

The data were tabulated and analyzed using the IBM SPSS Statistics version 24.0 software (SPSS Inc., Chicago, USA). Descriptive analyses were performed, and also a bivariate logistic regression, to verify the association between the use of SDF and the dentists' characteristics: workplace, experience, specialty, and city of professional practice. Statistical significance was set at $5 \%$, with a $95 \%$ confidence interval.

\section{Ethical Aspects}

The Human Research Ethics Committee of the Federal University of Rio de Janeiro approved the study (Protocol No. 1.604.702).

\section{Results}

A total of 409 dentists accessed and answered the questionnaire and were thus enrolled in this study. Most of the respondents worked in private offices (58.7\%), located in the capital (59.2\%), from which $22 \%$ were pediatric dentists, $20.9 \%$ orthodontists, and $15.7 \%$ general dentists. Considering dental specialties, $57.4 \%$ who reported using SDF were pediatric dentists, showing 6.76 times more chance to use SDF than other specialties. Dentists working at universities had 2.29 times more chance to use SDF than those working at private offices. Besides that, for each year of practice, the chance of using SDF increased by $3 \%$ (Table 1). Of the total, $13.2 \%$ of respondents used SDF, and 19.7\% reported not knowing of this product.

Table 1. Characteristics of dentists in RJ associated or not with the use of silver diamine fluoride.

\begin{tabular}{|c|c|c|c|c|c|}
\hline Variables & $\begin{array}{l}\text { Total } \\
\mathrm{N}(\%)\end{array}$ & $\begin{array}{c}\text { SDF Reported Usage } \\
\mathrm{N}(\%)\end{array}$ & OR & $95 \%$ CI & p-value \\
\hline \multicolumn{6}{|l|}{ Workplace } \\
\hline Private Office & $240(58.7)$ & $25(46.3)$ & 1.00 & & \\
\hline Public or Military Services & $93(22.7)$ & $13(24.1)$ & 1.40 & $0.68-2.86$ & 0.361 \\
\hline University & $76(18.6)$ & $16(29.6)$ & 2.29 & $1.15-4.57$ & $0.018^{*}$ \\
\hline \multicolumn{6}{|l|}{ City of Professional Practice } \\
\hline Capital & $242(59.2)$ & $33(61.1)$ & 1.00 & & \\
\hline Metropolitan Area & $43(10.5)$ & $2(3.7)$ & 0.31 & $0.71-1.33$ & 0.116 \\
\hline Other Cities & $78(19.1)$ & $10(18.5)$ & 0.93 & $0.43-1.98$ & 0.854 \\
\hline Missing Information & $46(11.2)$ & $9(16.7)$ & & & \\
\hline \multicolumn{6}{|l|}{ Speciality } \\
\hline Paediatric Dentists & $90(22.0)$ & $31(57.4)$ & 6.76 & $3.68-12.41$ & $<0.001 *$ \\
\hline Non-Paediatric Dentists & $319(78.0)$ & $23(42.6)$ & 1.00 & & \\
\hline \multicolumn{6}{|l|}{ Experience (Graduation Year) } \\
\hline Mean (SD) & $16.83(11.64)$ & $21.48(13.02)$ & 1.03 & $1.00-1.05$ & $0.008^{*}$ \\
\hline
\end{tabular}

OR: Odds Ratio; CI: Confidence Interval; *Statistically Significant.

Table 2 shows the indications of SDF pointed out by the dentists of RJ who reported to use SDF. Non-compliant patients $(75.9 \%)$, followed by care in places with no infrastructure $(68.5 \%)$, and patients with no capacity for collaboration (66.7\%), were the most cited clinical indications of SDF. Of those dentists that used SDF $(n=54), 75.9 \%$ indicated it only to primary dentition, and $24.1 \%$ in both primary and permanent dentition; 
$59.3 \%$ used it in anterior and posterior teeth, but $35.2 \%$ only used it in posterior teeth. Considering the type of caries lesion, $50 \%$ of dentists used SDF in enamel and dentin lesions, and one respondent reported the use of SDF without the presence of caries lesions (in any situation). There was no consensus about the application interval.

Table 2. Information about SDF usage.

\begin{tabular}{|c|c|}
\hline Indications of SDF Usage & $\begin{array}{c}\text { Use of SDF } \\
\mathrm{N}(\%)\end{array}$ \\
\hline \multicolumn{2}{|l|}{ Type of Lesion } \\
\hline Enamel Lesion & $8(14.8)$ \\
\hline Dentin Lesion & $18(33.3)$ \\
\hline Both Enamel and Dentin Lesion & $27(50)$ \\
\hline With or Without Lesion & $1(1.9)$ \\
\hline \multicolumn{2}{|l|}{ Type of Dentition } \\
\hline Only Primary Dentition & $41(75.9)$ \\
\hline Only Permanent Dentition & $\mathrm{O}(0.0)$ \\
\hline Both & $13(24.1)$ \\
\hline \multicolumn{2}{|l|}{ Type of Teeth } \\
\hline Anterior & $3(5.5)$ \\
\hline Posterior & $19(35.2)$ \\
\hline Both & $32(59.3)$ \\
\hline \multicolumn{2}{|l|}{ Clinical Situations* } \\
\hline Non-Compliant Patients & $41(75.9)$ \\
\hline No Capacity for Collaboration & $36(66.7)$ \\
\hline Elders & $10(18.5)$ \\
\hline Environmental Adequacy & $29(53.7)$ \\
\hline Lack of Infrastructure & $37(68.5)$ \\
\hline At High Risk of Caries & $28(51.9)$ \\
\hline \multicolumn{2}{|l|}{ Protocol - Application Interval } \\
\hline A Single Application & $13(24.1)$ \\
\hline 6-Month Reapplication & $11(20.4)$ \\
\hline Annual Reapplication & $\mathrm{O}(0.0)$ \\
\hline 4. Weekly Applications & $10(18.5)$ \\
\hline 4. Weekly Applications +6 Month Reapplication & $3(5.6)$ \\
\hline 4 Weekly Applications + Annual Reapplication & $4(7.4)$ \\
\hline A Different One & $12(22.2)$ \\
\hline Missing Information & $1(1.85)$ \\
\hline
\end{tabular}

The main barrier reported to the use of SDF, among those that did not use it, was a lack of scientific knowledge (58.3\%), followed by tooth darkening (27.6\%). Among those who used SDF, the barriers were tooth staining (90.7\%), and parental acceptance (64.8\%). Among respondents, 18.1\% were professors, and 59.5\% of them taught SDF to their students in theoretical classes, but only $28.4 \%$ used SDF in clinical classes (Table 3 ).

Table 3. Silver diamine fluoride (SDF) - barriers to use and teaching information.

\begin{tabular}{lccc}
\multicolumn{1}{c}{ Barriers } & Use of SDF & No & p-value \\
& N (\%) & N (\%) & \\
\hline $\begin{array}{l}\text { Possible Barriers to the Use of SDF } \\
\text { Parental Acceptance }\end{array}$ & $35(64.8)$ & $80(22.5)$ & $<0.001$ \\
Patient Acceptance & $8(14.8)$ & $39(11.0)$ & 0.411
\end{tabular}




\begin{tabular}{lccc} 
Scientific Knowledge & $11(20.4)$ & $207(58.3)$ & $<0.001$ \\
Inadequate Training & $3(5.6)$ & $97(27.3)$ & 0.001 \\
Reimbursement & $7(13.0)$ & $38(10.7)$ & 0.621 \\
Obtaining Product & $7(13.0)$ & $40(11.3)$ & 0.716 \\
Cost & $1(1.9)$ & $13(3.7)$ & 0.496 \\
Does not Restore Shape and Function & $19(35.2)$ & $36(10.1)$ & $<0.001$ \\
Tooth-Staining & $49(90.7)$ & $98(27.6)$ & $<.001$ \\
It Does not Arrest Caries & $2(3.7)$ & $335(81.9)$ & $<0.001$ \\
Teaching - Are You a Professor? & & $74(18.1)$ & $<0.001$ \\
No & $32(59.3)$ & $30(40.5)$ & $23(31.1)$ \\
Yes & $22(40.7)$ & $21(28.4)$ & \\
Teaching Practice & & & \\
Do not Teach & $0(0.0)$ & $3(13.6)$ & \\
Yes, Theoretical Classes & $19(86.4)$ & & \\
Yes, Theoretical and Clinical Classes & & & \\
\hline
\end{tabular}

\section{Discussion}

The use of SDF was associated with the workplace, experience, and specialty among dentists that participated in our survey. In this study, the authors observed that dentists working at universities had more than twice the chance to use SDF, compared to those that work in private offices; and the principal barrier to SDF use was a lack of knowledge. This may signal a disconnect between research and clinical practice. Generally, those who work at universities have quicker and easier access to new scientific evidence, which gives them knowledge and security in using new or underutilized, products, or techniques. Likewise, the greater the clinical experience, the more discerning and confident are the clinicians to employ these techniques.

Although SDF has been used for other clinical indications, such as tooth hypersensitivity, to prevent root caries in elderly people, and to sterilize infected root canals, the most employed clinical indications are the arrest of caries in the primary teeth of children, and prevention of pit and fissure caries in erupting permanent molars [9]. Therefore, we believe that this is the reason that pediatric dentist specialists are more likely to use SDF, as demonstrated by our survey.

Several decades are necessary to fully adopt new clinical practices [10], and for this to happen, it is necessary that the technique gains traction and confidence in dental communities. As SDF has been used in Brazil since the 1960s, a high adoption rate could be expected, but we found a low one among dentists in RJ, which is the second-largest economic state in Brazil. As in a previous study [10], we observed that less than one-third of the RJ dentistry professors used SDF in their clinical classes (Table 3). Aesthetic concerns added to a lack of scientific evidence, and the fact that there were no published Portuguese guidelines available [11], concerning the use of SDF, may be the explanation for this.

Among those who reported they used SDF, tooth staining and parental acceptance were the main barriers to use, as reported previously [10]. A recent review, however, questioned whether parental acceptance really was a barrier to SDF use, and concluded that there was insufficient evidence about the esthetic effects of SDF applications to primary teeth [12]. Actually, parents with limited options for treatment due to behavioral or medical limitations were more willing to accept SDF treatment [13]. It was previously suggested that dentists should emphasize to parents the ease of application of SDF, how it is child-friendly, and may even help to avoid treatments with physical restraint, or under general anesthesia [14]. This suggestion is based on their research, in which these authors observed that the negative feelings of the parents about tooth-staining decreased the more they understood the simplicity of the SDF technique. 
Confronting the information that those who work at universities have twice more chance to use DFP with the fact that the main barrier among respondents who do not use is the lack of scientific knowledge, we can see that there is a gap between scientific information produced in Universities and the clinical application by clinicians. Probably, this is the same reason for the absence of a consensus on the application interval for SDF treatment.

Herein, the most cited clinical indication for the use of SDF was 'behavioral issues'. Similarly, a survey [10] with pediatric dentistry residency program directors found that non-compliant patients were good candidates for treatment with SDF. Besides that, in both surveys, the majority of respondents disagreed that SDF should be used in permanent teeth when compared to primary teeth. This can be explained by the fact that SDF darkens the tooth, which is easier to accept in a temporary tooth, and because there is evidence that $\mathrm{SDF}$ is more effective in primary teeth than permanent teeth [6].

Interestingly, although lesions in posterior teeth had a lower chance of becoming arrested than anterior teeth $[15,16]$, more than one-third of the respondents in our survey reported the use of SDF only for posterior teeth, and $5.6 \%$ used it only for anterior teeth. It may be related to the fact that dentists still have prejudice to the use of SDF in anterior teeth due to aesthetic, and also to greater acceptability of the parents to the use of SDF in the posterior teeth when compared to the previous ones [13].

Since we delivered the present survey, SDF remains in the target of many studies that still seek to explore the best protocol, the perception, and acceptability of parents, the knowledge of professionals and their clinical efficacy [17-22]. Faced with the great interest in this product and its increasing use worldwide, surveys like ours becomes very important to evaluate the SDF status within each population.

\section{Conclusion}

SDF is not a commonly-used product, among the respondent dentists from RJ, to control dental caries. The teaching of SDF should be more widespread in undergraduate courses, and the scientific evidence should be better disseminated, and available in a simplified way, for access by clinicians.

\section{Authors' Contributions}

\begin{tabular}{|c|c|c|}
\hline ALV & (D) $0000-0003-0240-0107$ & $\begin{array}{l}\text { Conceptualization, Methodology, Formal Analysis, Writing - Original Draft } \\
\text { Preparation and Writing - Review and Editing. }\end{array}$ \\
\hline JPLM & (D) $0000-0003-1987-3584$ & Conceptualization, Methodology and Formal Analysis. \\
\hline RRL & (iD) $0000-0002-7784-9905$ & Conceptualization, Methodology and Formal Analysis. \\
\hline FBF & (iD) $0000-0002-4098-8637$ & Conceptualization, Methodology and Writing - Original Draft Preparation. \\
\hline $\mathrm{AFG}$ & (DD 0000-0001-6467-7078 & Conceptualization, Methodology and Writing - Original Draft Preparation. \\
\hline
\end{tabular}

\section{Financial Support}

Fundação de Amparo à Pesquisa do Estado do Rio de Janeiro - FAPERJ (Grant No. E-26/202.766/2019).

\section{Conflict of Interest}

The authors declare no conflicts of interest.

\section{References}

[1] World Health Organization. Oral health Available at: "http://www.who.int/mediacentre/factsheets/fs318/en/". [Accessed on June 15, 2017]. Archived by WebCite ${ }^{\circledR}$ at: "http://www.webcitation.org/6rFSmtXPZ". 
[2] Brasil. Ministério da Saúde. Secretaria de Atenção à Saúde. Secretaria de Vigilância em Saúde. SB Brasil 2010: Pesquisa Nacional de Saúde Bucal: Resultados Principais. Brasília: Ministério da Saúde, 2012. [In Portuguese].

[3] Edelstein BL. Solving the problem of early childhood caries: a challenge for us all. Arch Pediatr Adolesc Med 2009; 163(7):667-8. https://doi.org/10.1001/archpediatrics.2009.107

[4] Hobdell M, Petersen PE, Clarkson J, Johnson N. Global goals for oral health 2020. Int Dent J 2003; 53(5):285-8. https://doi.org/10.1111/j.1875-595x.2003.tbo0761.x

[5] Chu CH, Lo ECM, Lin HC. Effectiveness of silver diamine fluoride and sodium fluorid varnish in arresting dentin caries in Chinese pre-school children. J Dent Res 2002; 81(11):767-70. https://doi.org/10.1177/0810767

[6] Llodra JC, Rodriguez A, Ferrer B, Menardia V, Ramos T, Morato M. Efficacy of silver diamine fluoride for caries reduction in primary teeth and first permanent molars of schoolchildren: 36-month clinical trial. J Dent Res 2005; 84(8):72 1-4. https://doi.org/10.1177/154405910508400807

[7] Horst JA, Ellenikiotis H, Milgrom PL. UCSF protocol for caries arrest using silver diamine fluoride: rationale, indications, and consent. J Calif Dent Assoc 2016; 44(1):16-28.

[8] Ramos-Gomez FJ, Silva DR, Law CS, Pizzitola RL, John B, Crall JJ. Creating a new generation of pediatric dentists: a paradigm shift in training. J Dent Educ 2014; 78(12):1593-603.

[9] Zhao IS, Gao SS, Hiraishi N, Burrow MF, Duangthip D, Mei ML, et al. Mechanisms of silver diamine fluoride on arresting caries: a literature review Int Dent J 2018; 68(2):67-76. https://doi.org/10.1111/idj.12320

[10] Nelson T, Scott JM, Crystal YO, Berg JH, Milgrom P. Silver diamine fluoride in pediatric dentistry training programs: survey of graduate program directors. Pediatr Dent 2016; 38(3):212-7.

[11] American Academy of Pediatric Dentistry. Policy on the Use of Silver Diamine Fluoride for Pediatric Dental Patients. Pediatr Dent 2017; 39(6):51-3.

[12] Sousa FSO, Santos APP, Barja-Fidalgo F, Oliveira BH. Evidence-based pediatric dental practice within the clinician's reach: the case of the esthetic effect of topical silver diamine fluoride for caries control in primary dentition. Rev Gaúch Odontol 2016; 64(4):369-75.

[13] Crystal YO, Janal MN, Hamilton DS, Niederman R. Parental perceptions and acceptance of silver diamine fluoride staining. J Am Dent Assoc 2017; 148(7):510-8. https://doi.org/10.1016/j.adaj.2017.03.013

[14] Clemens J, Gold J, Chaffin J. Effect and acceptance of silver diamine fluoride treatment on dental caries in primary teeth. J Public Health Dent 2018; 78(1):63-8. https://doi.org/10.1111/jphd.12241

[15] Zhi QH, Lo EC, Lin HC. Randomized clinical trial on effectiveness of silver diamine fluoride and glass ionomer in arresting dentine caries in preschool children. J Dent 2012; 40(11):962-7. https://doi.org/10.1016/j.jdent.2012.08.002

[16] Fung MHT, Duangthip D, Wong MCM, Lo ECM, Chu CH. Arresting dentine caries with different concentration and periodicity of silver diamine fluoride. JDR Clin Trans Res 2016; 1(2):143-52. https://doi.org/10.1177/2380084416649150

[17] Burgette JM, Weintraub JA, Birken SA, Lewis TA, White BA. Development of a silver diamine fluoride protocol in safety net dental settings. J Dent Child 2019; 86(1):32-9.

[18] Crystal YO, Kreider B, Raveis VH. Parental expressed concerns about silver diamine fluoride (SDF) treatment. J Clin Pediatr Dent 2019; 43(3):155-60. https://doi.org/10.17796/1053-4625-43.3.2

[19] Trieu A, Mohamed A, Lynch E. Silver diamine fluoride versus sodium fluoride for arresting dentine caries in children: a systematic review and meta-analysis. Sci Rep 2019; 9(1):2115.

https://doi.org/10.1038/s41598-019-38569-9

[20] Antonioni MB, Fontana M, Salzmann LB, Inglehart MR. Pediatric dentists' silver diamine fluoride education, knowledge, attitudes, and professional behavior: a national survey. J Dent Educ 2019; 83(2):173-82. https://doi.org/10.21815/JDE.019.020

[21] Magno MB, Silva LPD, Ferreira DM, Barja-Fidalgo F, Fonseca-Gonçalves A. Aesthetic perception, acceptability and satisfaction in the treatment of caries lesions with silver diamine fluoride: a scoping review. Int J Paediatr Dent 2019; 29(3):257-66. https://doi.org/10.1111/ipd.12465

[22] Chibinski AC, Wambier LM, Feltrin J, Loguercio AD, Wambier DS, Reis A. Silver diamine fluoride has efficacy in controlling caries progression in primary teeth: a systematic review and meta-analysis. Caries Res 2017; 51(5):527-41. https://doi.org/10.1159/000478668 